\title{
Treatment Patterns and Predictors of Adherence in HIV Patients Receiving Single- or Multiple-Tablet Darunavir, Cobicistat, Emtricitabine, and Tenofovir Alafenamide
}

This article was published in the following Dove Press journal:

Patient Preference and Adherence

\author{
Wing Chow' \\ Prina Donga' \\ Aurélie Côté-Sergent ${ }^{2}$ \\ Carmine Rossi ${ }^{2}$ \\ Patrick Lefebvre ${ }^{2}$ \\ Marie-Hélène Lafeuille ${ }^{2}$ \\ Hélène Hardy ${ }^{2}$ \\ Bruno Emond ${ }^{3}$ \\ 'Real World Value \& Evidence, Janssen \\ Scientific Affairs, LLC, Titusville, NJ, USA; \\ ${ }^{2}$ Analysis Group, Inc., Montréal, QC, \\ Canada; ${ }^{3}$ Early Compound Development, \\ Janssen Research \& Development, LLC, \\ Titusville, NJ, USA
}

Purpose: Darunavir, cobicistat, emtricitabine, and tenofovir alafenamide can be used as a single-tablet regimen (STR, DRV/c/FTC/TAF) or multiple-tablet regimen (MTR, DRV/c + FTC/TAF) to treat patients with human immunodeficiency virus (HIV). This study described treatment patterns and predictors of adherence among patients with HIV initiated on DRV/c/ FTC/TAF or DRV/c+FTC/TAF.

Patients and Methods: A retrospective longitudinal study was conducted using linked claims and electronic medical records from Decision Resources Group's Real World Data Repository (7/ 17/2017-6/1/2019). Treatment-naïve and treatment-experienced virologically suppressed adults with HIV-1 prescribed DRV/c/FTC/TAF or DRV/c+FTC/TAF (index date) were included. Sixmonth persistence (no treatment gaps $>60$ and $>90$ days) and adherence (proportion of days covered [PDC]) to the index regimen were evaluated among patients with $\geq 6$ months of observation postindex. Predictors of low adherence ( $\mathrm{PDC}<80 \%$ ) were evaluated using a logistic regression model. Results: Among 2633 eligible patients (49.5 years old, 29\% female, 37\% African American/ Black), $12 \%$ were treatment-naïve pre-index and $88 \%$ switched from a previous antiretroviral therapy; $84 \%$ initiated DRV/c/FTC/TAF and 16\% initiated DRV/c+FTC/TAF. Among 822 DRV/ $\mathrm{c} / \mathrm{FTC} / \mathrm{TAF}$ patients with $\geq 6$ months of observation post-index, $80 \%$ and $86 \%$ had no $>60$ - and $>90$-day gaps in DRV/c/FTC/TAF coverage, respectively, while among 204 DRV/c+FTC/TAF patients with $\geq 6$ months of observation post-index, $69 \%$ and $75 \%$ had no $>60$ - and $>90$-day gaps in DRV/c+FTC/TAF coverage, respectively. Mean (median) PDC for the index regimen was $81 \%(93 \%)$ for patients treated with DRV/c/FTC/TAF and 73\% (83\%) for patients treated with $\mathrm{DRV} / \mathrm{c}+\mathrm{FTC} / \mathrm{TAF}$. Predictors of low adherence included younger age (odds ratio $[\mathrm{OR}]=2.36$, $\mathrm{p}=0.017)$, higher Quan-Charlson comorbidity index $(\mathrm{OR}=1.32, \mathrm{p}=0.012)$, use of MTR regimen at index $(\mathrm{OR}=1.69, \mathrm{p}=0.022)$, and prior low adherence $(\mathrm{OR}=2.56, \mathrm{p}<0.001)$.

Conclusion: Among patients initiating a DRV/c-based regimen, those initiating STR had higher 6-month adherence/persistence than those initiating MTR, highlighting the potential benefits of the STR formulation, particularly among younger patients with multiple comorbidities and prior low adherence.

Keywords: HIV, protease inhibitors, treatment adherence and compliance, patient compliance, administrative claims, healthcare, electronic health records

\section{Introduction}

While human immunodeficiency virus (HIV) remains ineradicable, the introduction of highly effective antiretroviral therapy (ART) has substantially improved the
Correspondence: $\mathrm{Br}$
Tel + I 5 I4-394-4455

Email Bruno.Emond@analysisgroup.com
Patient Preference and Adherence 2020:14 23I5-2326

2315 
prognosis of people living with HIV. ${ }^{1}$ However, ART use remains suboptimal and $<60 \%$ of HIV patients were reported to be virologically suppressed in 2016 in the United States (US). ${ }^{2,3}$ More recent estimates are available for smaller groups of patients which exceed the national viral suppression average, such as among those who received medical care from the Ryan White HIV/acquired immunodeficiency syndrome (AIDS) Program whereby $87 \%$ reached viral suppression in $2018 .{ }^{4}$ Nevertheless, a recent real-world study showed a mean proportion of days covered (PDC) of only $\sim 50 \%$ among Medicaid and commercially insured patients. ${ }^{5}$

The recommendation by consensus for patients with HIV was to achieve $\geq 95 \%$ adherence to ART. $^{6-9}$ Recently, the Pharmacy Quality Alliance, a nationally recognized organization which provides consensus-based measures for medication safety, adherence and appropriate use, recommended $\geq 90 \%$ adherence to be considered as optimal for ART. ${ }^{10}$ Suboptimal adherence to ART has been associated with an increased risk of virologic failure and drug resistance, resulting in a high burden for the healthcare system. ${ }^{7,11,12}$ The Department of Health and Human Services (DHHS) guidelines therefore recommend multiple strategies to improve adherence and persistence, including the use of tolerable and less complex ART regimens, such as single-table regimens (STRs). ${ }^{13}$ Among patients with adherence problems, the DHHS guidelines recommend using a darunavir (DRV)-based regimen because of its high genetic barrier to resistance. ${ }^{13}$ Approved on $1 / 29 / 2015$, DRV/cobicistat (DRV/c) is a boosted protease inhibitor (PI) which can be prescribed with emtricitabine/ tenofovir alafenamide (FTC/TAF) as a once daily multipletablet regimen (MTR) for the treatment of HIV-1 among treatment-naïve and treatment-experienced adults. On 7/17/ 2018, DRV/c/FTC/TAF $\left(\right.$ Symtuza $^{\circledR}$ ) became the first PIbased STR approved for HIV-1 treatment among treatmentnaïve patients and virologically suppressed adults stable on ART for $\geq 6$ months. ${ }^{14}$

While factors such as race, gender, and social determinants of health play an important role in adherence to ART, ${ }^{15-18}$ studies show that STRs confer benefits with regards to adherence ${ }^{5,19-27}$ and persistence ${ }^{25,28-30}$ compared to MTRs. However, whether DRV/c/FTC/TAF STR improves adherence and persistence compared to the 2-pill MTR formulation with the same components (ie DRV/c+FTC/TAF) remains to be assessed in a realworld setting. Therefore, this study aimed to describe the characteristics and treatment patterns, including adherence and persistence, of patients in the US insured through multiple payer channels and initiated on DRV/c-based STR or MTR, as well as identify predictors of adherence in these patients.

\section{Materials and Methods \\ Data Source}

The Decision Resources Group's (DRG) Real World Data Repository (7/17/2017-6/1/2019) was used for this study. The database is made up of open source claims data from multiple electronic data interchanges and electronic medical records (EMR) data from a major EMR vendor in the US. DRG's Real World Data Repository, which includes patients from all states in the US (including beneficiaries of the Acquired Immunodeficiency Syndrome Drug Assistance Program), is broadly representative of the entire US population. Of note, reasons for ART discontinuation are not available in the database. Data are de-identified and comply with the patient requirements of the Health Insurance Portability and Accountability Act (HIPAA). As this was an analysis of claims data, Institutional Review Board (IRB) approval was not required. Per Title 45 of the Code of Federal Regulations (CFR), Part 46.101(b)(4), ${ }^{31}$ the administrative claims data analysis of this study is exempt from the IRB review for two reasons: (1) it is a retrospective analysis of existing data (hence no patient intervention or interaction), (2) no patientidentifiable information is included in the claims dataset.

\section{Study Design}

A retrospective longitudinal study design was used (Figure 1). The index date was the date of initiation of DRV/c/FTC/TAF or $\mathrm{DRV} / \mathrm{c}+\mathrm{FTC} / \mathrm{TAF}$ on or after 7/17/2018. Priority was given to DRV/c/FTC/TAF followed by DRV/c+FTC/TAF when identifying the index date. For DRV/c+FTC/TAF, the index date was anchored at the date of the first claim for DRV/c, and FTC/TAF had to be claimed within \pm 14 days of the index date. The preindex period was the 12-month period of continuous clinical activity before the index date. The post-index period spanned from the index date until the earliest among end of continuous clinical activity or end of data availability. Continuous clinical activity was defined as a consecutive period of time where patients had $\geq 1$ claim in each 3-month interval. Since an intentto-treat approach was used, patients could switch to another ART regimen during the post-index period.

\section{Study Population}

Patients were included if they had $\geq 1$ claim for DRV on or after 7/17/2018, used DRV as part of an ART regimen with 
Index date (on or after 7/17/2018):

First day of data

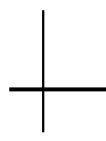

Date of first claim for DRV/C/FTC/TAF or $\mathrm{DRV} / \mathrm{c}$ (used in combination with FTC/TAF)
End of post-index period:

End of data availability $(6 / 1 / 2019)$ or end of continuous clinical activity

Figure I Study design scheme.

Abbreviations: c, cobicistat; DRV, darunavir; FTC, emtricitabine; TAF, tenofovir alafenamide.

c, FTC, and TAF (either DRV/c/FTC/TAF or DRV/c+FTC/ TAF), had $\geq 12$ months of continuous clinical activity preindex, $\geq 1$ claim with a diagnosis for HIV-1 on or before the index date, and were $\geq 18$ years old on the index date. Patients were considered treatment-naïve if they had no claim for ART during the pre-index period, while ART switchers had $\geq 1$ claim for ART during the pre-index period. Patients were excluded if they had history of ART and were not virologically suppressed during the 6-month period pre-index (ie $\geq 1$ viral load test result $\geq 50$ copies/mL during the 6-month period pre-index; viral load test results were obtained from EMR data), or had $\geq 1$ claim with a diagnosis for HIV-2, liver disease, chronic renal insufficiency, pregnancy, or cancer during the 12month pre-index period.

\section{Study Measures}

Demographic and clinical characteristics were described during the 12-month pre-index period. Treatment patterns were evaluated pre-index and over the post-index period (including switch to another ART). Among patients with $\geq 6$ months of continuous clinical activity post-index, persistence to the index regimen was evaluated during the first 6 months post-index using the proportion of patients with no gap $>60$ and $>90$ days in index regimen coverage. For patients initiated on DRV/c+FTC/TAF, both DRV/c and FTC/TAF had to be taken to be considered persistent. If one of the medications from the regimen was missing, the patient was considered to have a gap in treatment. If one of the medications from the regimen was missing for $>60$ or $>90$ days, the patient was considered non-persistent.

Among patients with $\geq 6$ months of continuous clinical activity post-index and $\geq 1$ ART claim during the 6 months pre- and post-index, adherence to the index regimen during the 6 months post-index and adherence to any ART during the 6 months pre- and post-index were evaluated. Adherence to the index regimen was measured using PDC, defined as the sum of non-overlapping days of supply for the index regimen divided by 6 months. The following PDC thresholds were reported: $\mathrm{PDC}<80 \%$ (nonadherence), $\mathrm{PDC} \geq 80 \%$ and $<95 \%$ (suboptimal adherence), $\mathrm{PDC} \geq 95 \%$ (optimal adherence). Additionally, given the recent change in the recommended adherence threshold, ${ }^{10}$ PDC $\geq 90 \%$ was also reported. For DRV/c + FTC/TAF, PDC by the index regimen was defined as the sum of non-overlapping days during which patients had a simultaneous supply of DRV/c and FTC/TAF. ${ }^{32}$

\section{Statistical Analysis}

Pre-index demographic and clinical characteristics as well as treatment patterns (including adherence and persistence) were descriptively evaluated using means, standard deviations (SDs), and medians for continuous variables, and frequencies and proportions for categorical variables. A multivariable logistic regression model was used to evaluate pre-index predictors of low adherence to the index regimen, defined as PDC for the index regimen $<80 \%$ at 6 months. Results were reported as odds ratios (ORs) with 95\% confidence intervals and p-values.

\section{Results}

Among 846,574 patients with $\geq 1$ claim with an HIV-1 diagnosis, 2633 were included in the study. A total of 308 $(12 \%)$ patients were treatment-naïve pre-index and 2325 (88\%) switched from a previous ART (Figure 2). At the index date, $2218(84 \%)$ and $415(16 \%)$ patients initiated $\mathrm{DRV} / \mathrm{c} / \mathrm{FTC} / \mathrm{TAF}$ and $\mathrm{DRV} / \mathrm{c}+\mathrm{FTC} / \mathrm{TAF}$, respectively. 


\begin{tabular}{|c|c|}
\hline $\begin{array}{l}\geq 1 \text { claim with a diagnosis of HIV-1 } \\
\text { (ICD-9 CM codes: 042, 795.71 and V08; ICD-10 CM codes: B20, R75, an } \\
\qquad \mathbf{N}=\mathbf{8 4 6 , 5 7 4}\end{array}$ & Z21) \\
\hline$\downarrow$ & \\
\hline $\begin{array}{l}\text { Initiation of darunavir on or after } 7 / 17 / 2018 \text {, defined as the index dat } \\
\qquad \mathrm{N}=9094(\mathbf{1 . 1 \% )}\end{array}$ & \\
\hline$\downarrow$ & \\
\hline $\begin{array}{l}\text { Use of darunavir as part of an ART regimen with cobicistat, FTC and T } \\
\qquad \mathbf{N}=\mathbf{5 4 1 8}(\mathbf{5 9 . 6 \% )}\end{array}$ & \\
\hline$\downarrow$ & \\
\hline $\begin{array}{l}\geq 12 \text { months of continuous clinical activity before the index date (pre-index } \\
\qquad N=3518(64.9 \%)\end{array}$ & eriod) \\
\hline$\downarrow$ & \\
\hline $\begin{array}{l}\geq 1 \text { claim with a diagnosis for HIV-1 on or before the index date } \\
\qquad \mathbf{N}=\mathbf{3 4 3 7}(\mathbf{9 7 . 7} \%)\end{array}$ & \\
\hline$\downarrow$ & \\
\hline $\begin{array}{l}\geq 18 \text { years old as of the index date } \\
\qquad \mathbf{N}=\mathbf{3 4 3 4}(\mathbf{9 9 . 9 \% )}\end{array}$ & \\
\hline$\downarrow$ & \\
\hline Exclusion criteria: & \\
\hline Patients who are not virologically suppressed during the 6-month period pre-index & $N=6(0.2 \%)$ \\
\hline$\geq 1$ claim with a diagnosis for HIV-2 during the pre-index period & $N=26(0.8 \%)$ \\
\hline $\begin{array}{l}\geq 1 \text { claim with a diagnosis for liver disease, including cirrhosis or hepatitis during the pre- } \\
\text { index period }\end{array}$ & $\mathrm{N}=484(14.1 \%)$ \\
\hline$\geq 1$ claim with a diagnosis for chronic renal insufficiency during the pre-index period & $\mathrm{N}=202(5.9 \%)$ \\
\hline$\geq 1$ claim with a diagnosis for pregnancy during the pre-index period & $N=19(0.6 \%)$ \\
\hline $\begin{array}{l}\geq 1 \text { claim with a diagnosis for cancer, excluding cutaneous Kaposi's sarcoma, basal cell } \\
\text { carcinoma, or resected, non-invasive cutaneous squamous carcinoma during the pre- } \\
\text { index period }\end{array}$ & $N=182(5.3 \%)$ \\
\hline Patients using DRV, c, FTC, and TAF in $\geq 3$-pill MTR & $N=5(0.1 \%)$ \\
\hline$\downarrow$ & \\
\hline $\begin{array}{l}\text { Patients eligible for the study } \\
\qquad \mathbf{N}=2633(\mathbf{7 6 . 7 \% )}\end{array}$ & \\
\hline Treatment-naïve patients $^{a}$ & $N=308(11.7 \%)$ \\
\hline Patients who switched from a previous ART to the index regimen ${ }^{b}$ & $\mathrm{~N}=2325(88.3 \%)$ \\
\hline
\end{tabular}

Figure 2 Identification of the study population.

Notes: ${ }^{a}$ Patients with no claims for an antiretroviral prior to the index date. ${ }^{b}$ Patients with $\geq 1$ claim for an antiretroviral prior to the index date.

Abbreviations: ART, antiretroviral therapy; c, cobicistat; DRV, darunavir; FTC, emtricitabine; HIV, human immunodeficiency virus; ICD-9 CM/ICD-I0 CM, International Classification of Disease, Ninth/Tenth Revision, Clinical Modification; MTR, multiple-tablet regimen; TAF, tenofovir alafenamide.

Overall, patients were observed for an average of 148 days $(\mathrm{SD}=85)$ post-index.

\section{Pre-Index Characteristics}

During the 12-month pre-index period, mean age was 49.5 years and 29\% were female (Table 1). Among 795 (30\%) patients with EMR data, 37\% were African American/ Black; $60 \%$ commercially insured, $20 \%$ covered by Medicare, and $16 \%$ covered by Medicaid. The 5 states with the highest proportions of patients were New York
(19\%), Florida (13\%), Texas (8\%), California (8\%), and Michigan (6\%). The mean time from first HIV diagnosis to index date was 53.6 months among patients with available information on HIV disease onset $(\mathrm{N}=196$ [7\%]). The mean Quan-Charlson comorbidity index (CCI) score $^{33}$ (excluding HIV symptoms) was 0.5. Hypertension (30\%), substance-related and addictive disorders (24\%), depression $(17 \%)$, chronic pulmonary disease $(17 \%)$, and anxiety (13\%) were commonly observed comorbidities. Characteristics were similar for patients initiated on DRV/ 
c/FTC/TAF (mean age: 49.7 years; 28.8\% female; 36.9\% African American/Black) and DRV/c+FTC/TAF (mean age: 48.5 years; $33.0 \%$ female; $38.3 \%$ African American/ Black).

\section{Treatment Patterns}

During the pre-index period, $72 \%$ of the 2633 patients received a protease inhibitor (PI)-based regimen, including $64 \%$ who received a DRV-based regimen; $32 \%$ received an integrase strand transfer inhibitor (INSTI)-based regimen, $7 \%$ received a non-nucleoside reverse transcriptase inhibitor (NNRTI)-based regimen, and $57 \%$ had TAF as a component in their ART regimen (Figure 3). During the post-index period, $25 \%$ of patients switched to an INSTI-based regimen and $4 \%$ switched to an NNRTIbased regimen following the index regimen. The proportion of patients using STR during the pre-index period was $15 \%$, and increased to $86 \%$ on or following the index date. The proportion of patients using MTR during the preindex period was $69 \%$, and decreased to $43 \%$ on or following the index date.

\section{Persistence to Index ART}

Among 1026 patients with $\geq 6$ months of continuous clinical activity post-index, $78 \%$ had no gap in index regimen coverage for $>60$ days and $84 \%$ had no gap in index regimen coverage for $>90$ days (Figure 4A). Persistence was higher among DRV/c/FTC/TAF users than DRV/c+FTC/TAF users, as seen by the larger proportion of $\mathrm{DRV} / \mathrm{c} / \mathrm{FTC} / \mathrm{TAF}$ users without coverage gaps $>60$ days $(\mathrm{DRV} / \mathrm{c} / \mathrm{FTC} / \mathrm{TAF}=80 \%$ and $\mathrm{DRV} / \mathrm{c}+\mathrm{FTC} / \mathrm{TAF}=69 \%)$ and $>90$ days $(\mathrm{DRV} / \mathrm{c} / \mathrm{FTC} /$ $\mathrm{TAF}=86 \%$ and $\mathrm{DRV} / \mathrm{c}+\mathrm{FTC} / \mathrm{TAF}=75 \%$ ).

\section{Adherence to Index ART Regimen and to Any ARTs}

Among 867 patients with $\geq 6$ months of continuous clinical activity post-index and $\geq 1$ ART claim during the 6 months pre- and post-index, $86 \%$ were initiated on $\mathrm{DRV} / \mathrm{c} / \mathrm{FTC} / \mathrm{TAF}$ and $14 \%$ on DRV/c+FTC/TAF. Mean (median) PDC by index ART was 81\% (93\%) among DRV/c/FTC/TAF users and $73 \%(83 \%)$ among DRV/c+FTC/TAF users post-index. Non-adherence (ie PDC $<80 \%$ ) was more common among DRV/c+FTC/TAF users (46\%) than DRV/c/FTC/TAF users (28\%; Figure 4B). Optimal adherence (ie PDC $\geq 95 \%$ ) was more common among DRV/c/FTC/TAF users (46\%) than DRV/c+FTC/TAF users (34\%). Similarly, the proportion of
Table I Demographic and Clinical Characteristics in the 12 Month Pre-Index Period

\begin{tabular}{|c|c|}
\hline \multirow[t]{2}{*}{ Patient Characteristics } & All Patients \\
\hline & $N=2633$ \\
\hline Age at index date (years), mean \pm SD [median] & $\begin{array}{l}49.5 \pm 11.8 \\
{[51.0]}\end{array}$ \\
\hline Female, n (\%) & $775(29.4)$ \\
\hline $\begin{array}{l}\text { Patients in EMR data, n (\%) } \\
\text { Race, n (\%) } \\
\text { African American/Black } \\
\text { White } \\
\text { Hispanic } \\
\text { Other } \\
\text { Unknown }\end{array}$ & $\begin{array}{l}295(37.1) \\
190(23.9) \\
31(3.9) \\
6(0.8) \\
273(34.3)\end{array}$ \\
\hline $\begin{array}{l}\text { US geographic region }{ }^{\mathrm{a}} \text {, n (\%) } \\
\text { South } \\
\text { Florida } \\
\text { Texas } \\
\text { Northeast } \\
\text { New York } \\
\text { Midwest } \\
\text { Michigan } \\
\text { West } \\
\text { California } \\
\text { Unknown }\end{array}$ & $\begin{array}{l}1135(43.1) \\
339(12.9) \\
213(8.1) \\
666(25.3) \\
489(18.6) \\
388(14.7) \\
155(5.9) \\
387(14.7) \\
202(7.7) \\
57(2.2)\end{array}$ \\
\hline $\begin{array}{l}\text { Insurance plan/payer type }{ }^{\mathrm{a}}, \mathrm{n}(\%) \\
\text { Commercial plans } \\
\text { Medicare } \\
\text { Medicaid } \\
\text { Other } \\
\text { Unknown }\end{array}$ & $\begin{array}{l}1574(59.8) \\
526(20.0) \\
426(16.2) \\
68(2.5) \\
39(1.5)\end{array}$ \\
\hline $\begin{array}{l}\text { Year of index date, n (\%) } \\
2018 \\
2019\end{array}$ & $\begin{array}{l}1274(48.4 \%) \\
1359(51.6 \%)\end{array}$ \\
\hline $\begin{array}{l}\text { Patients with HIV disease onset information in } \\
\text { EMR data, } n(\%) \\
\text { Time (in months) between HIV disease onset } \\
\text { and index date, mean } \pm \text { SD [median] }\end{array}$ & $\begin{array}{l}196(7.4) \\
53.6 \pm 77.0 \\
{[28.2]}\end{array}$ \\
\hline $\begin{array}{l}\text { Comorbidities, } \mathrm{n}(\%) \\
\text { Hypertension } \\
\text { Substance-related and addictive disorders } \\
\text { Depression } \\
\text { Chronic pulmonary disease } \\
\text { Drug abuse } \\
\text { Anxiety disorders } \\
\text { Obesity } \\
\text { Type II diabetes } \\
\text { Psychoses } \\
\text { Opportunistic infections }\end{array}$ & $\begin{array}{l}778(29.5) \\
624(23.7) \\
456(17.3) \\
456(17.3) \\
370(14.1) \\
353(13.4) \\
315(12.0) \\
301(11.4) \\
293(11.1) \\
61(2.3)\end{array}$ \\
\hline
\end{tabular}

(Continued) 
Table I (Continued).

\begin{tabular}{|l|l|}
\hline Patient Characteristics & All Patients \\
\cline { 2 - 2 } & $\mathbf{N}=\mathbf{2 6 3 3}$ \\
\hline Quan-CCl, mean \pm SD [median] & $4.7 \pm 3.1[6.0]$ \\
Quan-CCl (excluding HIV symptoms), mean \pm SD & $0.5 \pm 1.0[0.0]$ \\
[median] & \\
\hline
\end{tabular}

Note: ${ }^{a}$ Evaluated on the date closest to the index date.

Abbreviations: $\mathrm{CCl}$, Charlson comorbidity index; EMR, electronic medical records; HIV, human immunodeficiency virus; SD, standard deviation; US, United States.

patients with $\mathrm{PDC} \geq 90 \%$ was higher among DRV/c/FTC/ TAF users (58\%) than DRV/c+FTC/TAF users (45\%).

Among the 867 patients identified for the evaluation of PDC, mean (median) PDC by any ART increased from $77 \%$ (86\%) during the 6-month pre-index period to $89 \%(97 \%)$ during the 6-month post-index period, with most of the observed PDC post-index being associated with the use of the index regimen (ie DRV/c/FTC/TAF or DRV/c+FTC/TAF; mean [median] PDC by index ART=80\% [93\%]). The proportion of patients with a PDC $<80 \%$ by any ART decreased from $36 \%$ during the 6 -month pre-index period to $17 \%$ during the 6-month post-index period, the proportion of patients with a PDC $\geq 80 \%$ and $<95 \%$ decreased from $43 \%$ to $26 \%$, and the proportion of patients with a $\mathrm{PDC} \geq 95 \%$ increased from $21 \%$ to $57 \%$. Similarly, the proportion of patients with $\mathrm{PDC} \geq 90 \%$ increased from $38 \%$ pre-index to $69 \%$ post-index.

\section{Predictors of Low Adherence}

The odds of non-adherence were significantly higher among patients aged 18-34 years (adjusted $\mathrm{OR}=2.36$, $\mathrm{p}=0.017$ ), patients residing in the Northeast region (adjusted $\mathrm{OR}=1.98, \mathrm{p}<0.001$ ), patients with a higher Quan$\mathrm{CCI}$ (adjusted $\mathrm{OR}=1.32, \mathrm{p}=0.012$ ), patients initiated on an MTR at the index date (adjusted $\mathrm{OR}=1.69, \mathrm{p}=0.022$ ), and patients with a PDC to any ART $<80 \%$ during the 6 months pre-index (adjusted $\mathrm{OR}=2.56, \mathrm{p}<0.001$; Figure 5).

\section{Discussion}

This study is the first to assess real-world persistence and adherence associated with DRV/c/FTC/TAF or DRV/c + FTC/TAF. Included patients represented a diverse population (ie 37\% African American/Black, 29\% females, 16\% Medicaid) with various comorbidities. The majority of patients used DRV/c/FTC/TAF and had previously received

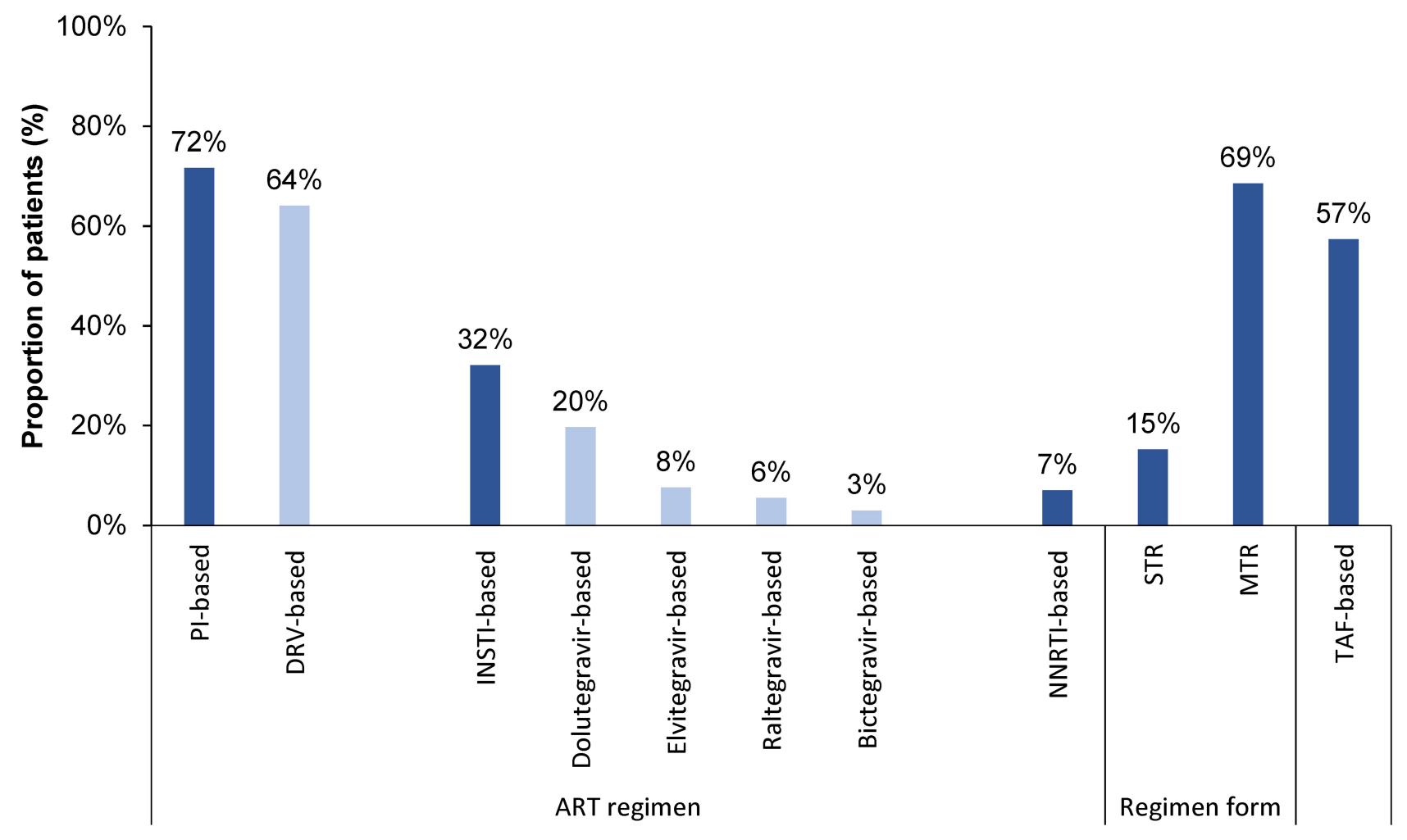

Figure 3 ART regimens received during the 12-month pre-index period.

Abbreviations: ART, antiretroviral therapy; DRV, darunavir; INSTI, integrase strand transfer inhibitor; MTR, multiple-tablet regimen; NNRTI, non-nucleoside reverse transcriptase inhibitor; PI, protease inhibitor; STR, single-tablet regimen; TAF, tenofovir alafenamide. 


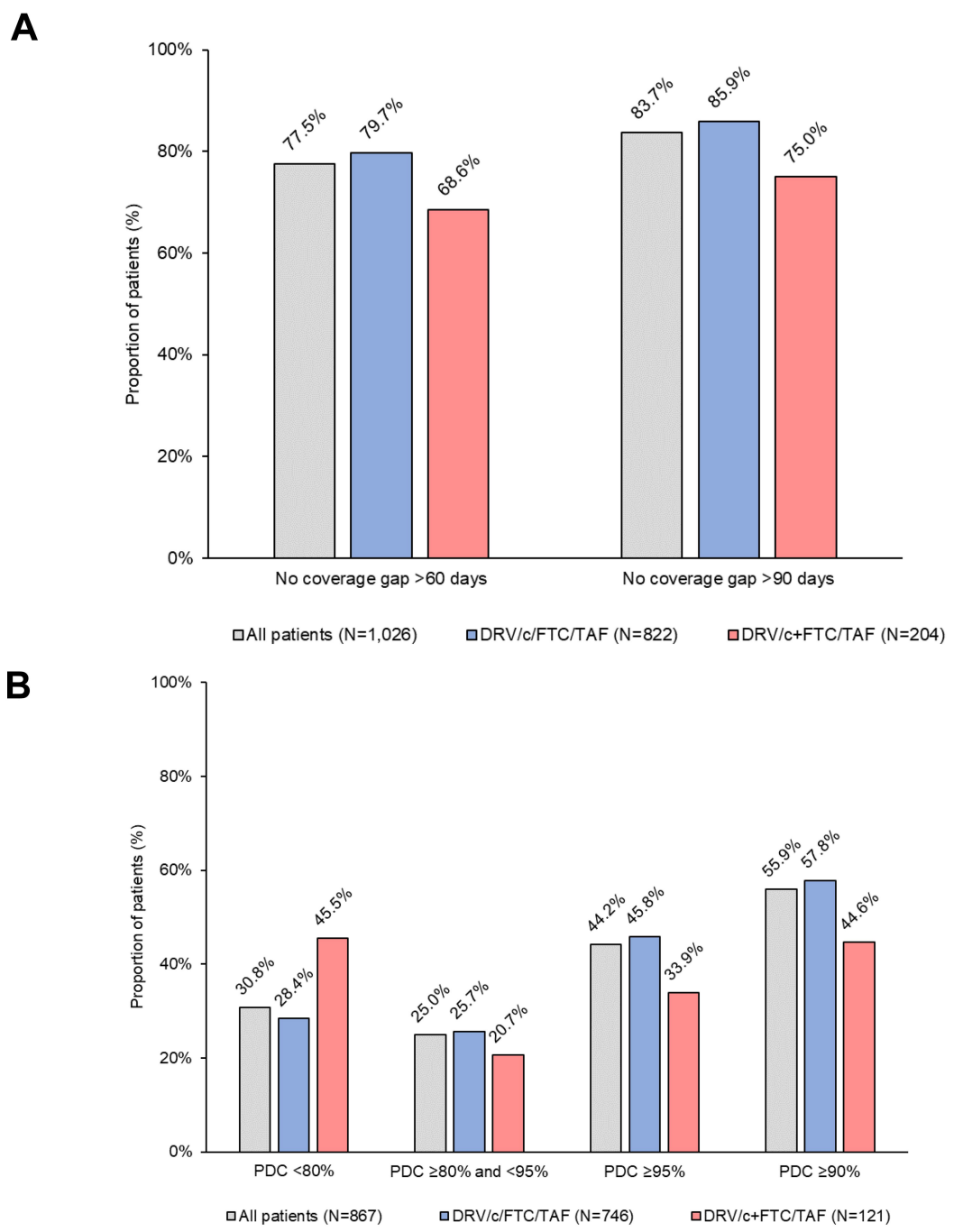

\begin{tabular}{lccc}
\hline & All patients & DRV/c/FTC/TAF & DRV/c+FTC/TAF \\
\hline $\begin{array}{l}\text { PDC by index ART, } \\
\%, \text { mean } \pm \text { SD } \\
\text { [median] }\end{array}$ & $80 \pm 26[93]$ & $81 \pm 26[93]$ & $73 \pm 29[83]$ \\
\hline
\end{tabular}

Figure 4 (A) Persistence and (B) adherence to the index ART regimen during the first 6 months post-index.

Abbreviations: ART, antiretroviral therapy; c, cobicistat; DRV, darunavir; FTC, emtricitabine; PDC, proportion of days covered; SD, standard deviation; TAF, tenofovir alafenamide.

another ART. On average, patients initiated treatment 53.6 months (4.5 years) after their first HIV diagnosis.

Patients using STR had higher persistence and adherence to the index regimen than those using MTR, consistent with prior studies. ${ }^{25,28-30}$ Regardless of the drug formulation, persistence rates remained high 6 months after the index date, corroborating adherence results for treatment-naïve patients rapidly initiated on DRV/c/FTC/ TAF in the DIAMOND study, where mean (median) adherence as measured by pill count was 95\% (99\%) over a 48 -week study period. ${ }^{34}$ Post-index, a higher proportion of patients achieved $\mathrm{PDC} \geq 95 \%$ or $\mathrm{PDC} \geq 90 \%$ with
$\mathrm{DRV} / \mathrm{c} / \mathrm{FTC} / \mathrm{TAF}$ than with DRV/c+FTC/TAF, consistent with previous literature showing that adherence is higher with STRs than MTRs, ${ }^{5,19-27,30,35}$ even when the components of the regimens are the same, as is the case in the current study (2-pill MTR versus STR). Additionally, $>50 \%$ of patients achieved PDC to any ART $\geq 95 \%$, higher than the $14-20 \%$ who achieved this threshold in a previous study. ${ }^{5}$ Given the known association between optimal adherence and lower rates of drug resistance and virologic failure $^{7}$, the results of this study suggest that DRV/c/FTC/ TAF may help decrease these risks. ${ }^{11,12}$ The proportion of patients achieving PDC to any ART $\geq 95 \%$ at 6 months 


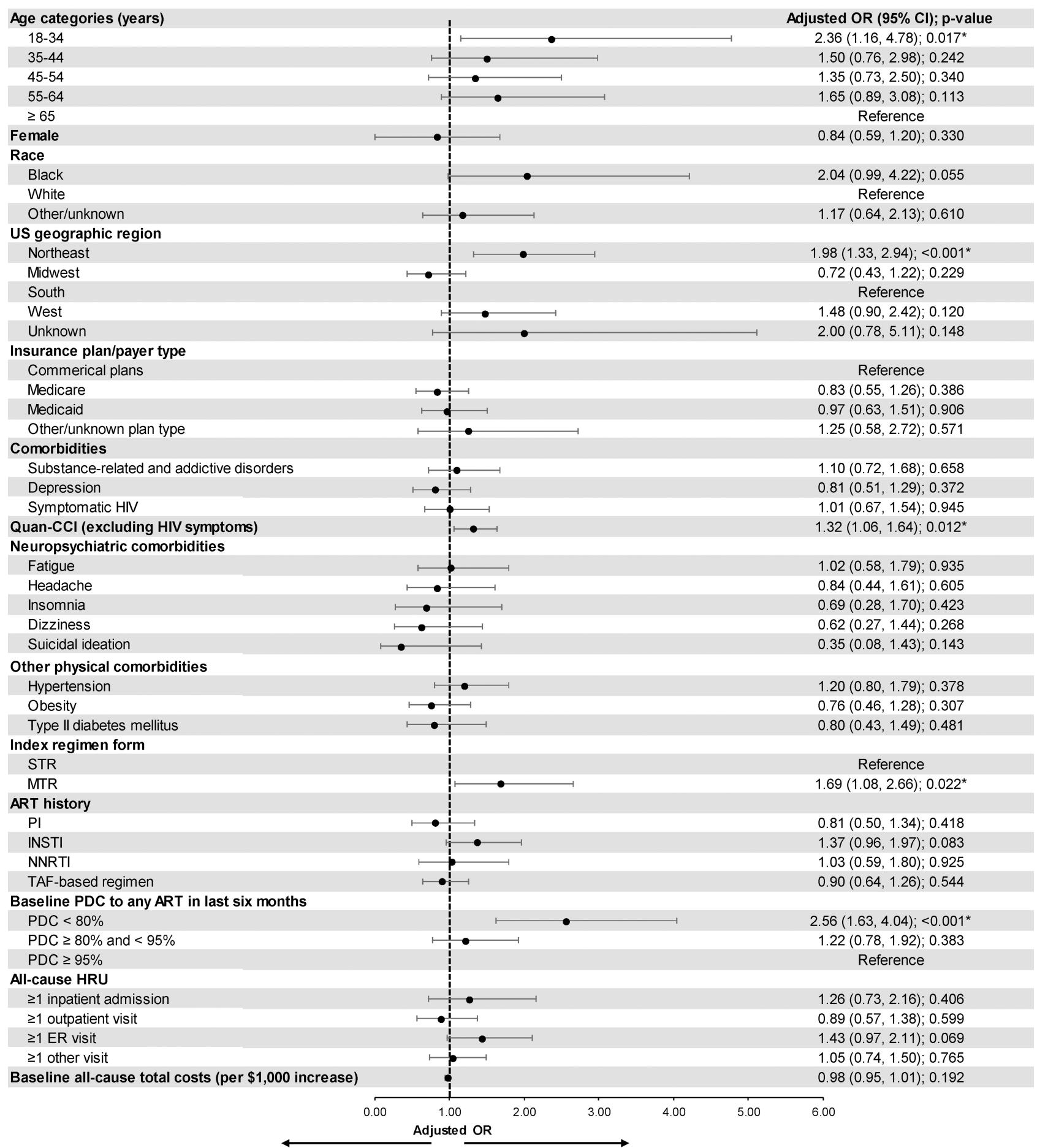

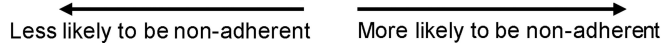

Figure 5 Predictors of low adherence.

Abbreviations: ART, antiretroviral therapy; $\mathrm{Cl}$, confidence interval; ER, emergency room; HIV, human immunodeficiency virus; HRU, healthcare resource utilization; INSTI, integrase strand transfer inhibitor; MTR, multiple-tablet regimen; NNRTI, non-nucleoside reverse transcriptase inhibitor; OR, odds ratio; PDC, proportion of days covered; PI, protease inhibitor; CCl, Charlson comorbidity index; STR, single-tablet regimen; TAF, tenofovir alafenamide; US, United States.

substantially increased from pre- to post-index $(21 \%$ vs $57 \%$ ). While many demographic factors may impact adherence, evidence from prior published work suggests that one challenge that may prevent a higher proportion of patients from reaching $\mathrm{PDC} \geq 95 \%$ is weight gain, which has been associated with some ART regimens such as INSTIs $^{36-39}$ (in the current study, 32\% of patients previously received an INSTI-based ART regimen). Weight 
gain has been shown to further compound non-adherence issues $^{40}$ and lead to an increased risk of diabetes and cardiovascular disease. ${ }^{41}$

Predictors of non-adherence were aligned with previous literature. Younger patients aged 18-34 years were more likely to be non-adherent to their index ART regimen than older patients, similar to a previous study. ${ }^{16}$ African American/Black patients were also associated with a strong - though borderline statistically non-significant increase in the odds of non-adherence compared with White patients $(\mathrm{OR}=2.04, \mathrm{p}=0.055)$. This result validates a previous study in which race was associated with a near-significant difference in self-reported adherence to ART $(\mathrm{p}=0.0558),{ }^{15}$ and another which reported the odds of $100 \%$ medication adherence were 40\% lower among African Americans/Black patients compared to White patients. ${ }^{17}$ Furthermore, the observation that patients with a higher Quan-CCI had higher odds of non-adherence is consistent with a previous study showing that the use of medications for other comorbidities in addition to ART was a predictor of ART non-adherence. ${ }^{42}$ Together, our data suggest that greater consideration for STRs should be placed among the younger, non-White patients (representing the majority of new HIV diagnoses in the US $)^{43}$ who have multiple comorbidities. The multivariable analysis also confirmed that receiving DRV/c+FTC/TAF was associated with significantly higher odds of nonadherence than receiving DRV/c/FTC/TAF. Other factors that could potentially impact adherence and persistence to ART but could not be assessed in this claims database are social and behavioral traits.

This study is subject to certain limitations. First, it was descriptive in nature and did not adjust for potential differences in characteristics between patients initiated on $\mathrm{DRV} / \mathrm{c} / \mathrm{FTC} / \mathrm{TAF}$ or DRV/c+FTC/TAF. Indeed, some differences may exist between the two patient groups which could influence their adherence and persistence to ART. Second, DRG is a provider-based data source that does not capture the services patients received from a provider that is outside of the network. However, even if patients change providers to obtain different medications, they tend to obtain all claims for the same medication through a single provider. Third, as with all claims data sources, DRG's Real World Data Repository may contain inaccuracies or omissions in diagnoses, billing, and other variables. Fourth, since the study was conducted on US patients, the results may not be generalizable to patients in other countries as there may be differences in patient characteristics, treatment practices, and healthcare systems. Finally, ART claims are assumed to indicate their use; however, patients may not adhere to the treatment regimen as prescribed.

\section{Conclusion}

In the current study, $>85 \%$ of patients were treatmentexperienced and switched from a previous ART. Adherence to any ART increased following the initiation of $\mathrm{DRV} / \mathrm{c} / \mathrm{FTC} / \mathrm{TAF}$ or $\mathrm{DRV} / \mathrm{c}+\mathrm{FTC} / \mathrm{TAF}$ and 6-month persistence and adherence were higher for the STR DRV/ c/FTC/TAF than for the 2-pill MTR DRV/c+FTC/TAF. Predictors of low adherence included younger age, higher Quan-CCI, initiation of an MTR regimen on the index date, and low adherence to previous ART regimens. Further research with longer follow-up period is needed to confirm persistence and adherence trends over time.

\section{Abbreviations}

ART, antiretroviral therapy; c, cobicistat; CCI, Charlson comorbidity index; CFR, Code of Federal Regulations; CI, confidence interval; DHHS, US Department of Health and Human Services; DRG, Decision Resources Group; DRV, darunavir; EMR, electronic medical record; ER, emergency room; FTC, emtricitabine; HIPAA, Health Insurance Portability and Accountability Act; HIV, human immunodeficiency virus; HRU, healthcare resource utilization; ICD-9 CM, International Classification of Diseases, 9th Revision, Clinical Modification; ICD-10 CM, International Classification of Diseases, 10th Revision, Clinical Modification; INSTI, integrase strand transfer inhibitor; IRB, Institutional Review Board; MTR, multi-tablet regimen; NNRTI, non-nucleoside reverse transcriptase inhibitors; OR, odds ratio; PDC, proportion of days covered; PI, protease inhibitor; SD, standard deviation; STR, single-tablet regimen; TAF, tenofovir alafenamide; US, United States.

\section{Data Sharing Statement}

The data that support the findings of this study are available from DRG but restrictions apply to the availability of these data, which were used pursuant to a data use agreement. The data are available through requests made directly to $\mathrm{DRG}$, subject to DRG's requirements for data access.

\section{Ethics Approval and Informed Consent}

Data are de-identified and comply with the patient requirements of the HIPAA. As this was an analysis of claims data, 
IRB approval was not required. Per Title 45 of the CFR, Part 46.101(b)(4), ${ }^{31}$ the administrative claims data analysis of this study is exempt from the IRB review for two reasons: (1) it is a retrospective analysis of existing data (hence no patient intervention or interaction), (2) no patientidentifiable information is included in the claims dataset.

\section{Consent for Publication}

All authors confirm that the contents of this manuscript can be published.

\section{Acknowledgments}

Medical writing assistance was provided by Samuel Rochette, MSc, and Loraine Georgy, PhD, employees of Analysis Group, Inc., a consulting company that has provided paid consulting services to Janssen Scientific Affairs, LLC, which funded the development and conduct of this study and manuscript.

\section{Author Contributions}

All authors made a significant contribution to the work reported, whether that is in the conception, study design, execution, acquisition of data, analysis and interpretation, or in all these areas; took part in drafting, revising or critically reviewing the article; gave final approval of the version to be published; have agreed on the journal to which the article has been submitted; and agree to be accountable for all aspects of the work.

\section{Funding}

This study was supported by Janssen Scientific Affairs, LLC. The sponsor was involved in the study design, interpretation of results, manuscript preparation, and publication decisions.

\section{Disclosure}

Dr Wing Chow is an employee of Janssen Scientific Affairs, LLC, during the conduct of the study; and owns stocks/shares at Johnson \& Johnson, outside the submitted work. Ms Prina Donga is an employee of Janssen Scientific Affairs, LLC. Ms Aurélie Côté-Sergent is an employee of Analysis Group, Inc., a consulting company that has provided paid consulting services to Janssen Scientific Affairs, LLC, which funded the development and conduct of this study and manuscript. Dr Carmine Rossi is an employee of Analysis Group, Inc., a consulting company that has provided paid consulting services to Janssen Scientific Affairs, LLC, which funded the development and conduct of this study and manuscript. $\mathrm{Mr}$ Patrick Lefebvre is an employee of Analysis Group, Inc., a consulting company that has provided paid consulting services to Janssen Scientific Affairs, LLC, which funded the development and conduct of this study and manuscript, and funded additional HIV researches in the past 36 months. Ms Marie-Hélène Lafeuille is an employee of Analysis Group, Inc., a consulting company, that has provided paid consulting services to Janssen Scientific Affairs, LLC to conduct this study; and has provided paid consulting services to Pharmacyclics and GlaxoSmithKline, outside the submitted work. Dr Hélène Hardy is an employee of Johnson and Johnson. Mr Bruno Emond is an employee of Analysis Group, Inc., a consulting company that has provided paid consulting services to Janssen Affairs, LLC, which funded the development and conduct of this study and manuscript. The authors report no other conflicts of interest in this work.

\section{References}

1. Antiretroviral Therapy Cohort Collaboration. Survival of HIV-positive patients starting antiretroviral therapy between 1996 and 2013: a collaborative analysis of cohort studies. Lancet HIV. 2017;4(8):e349-e356. doi:10.1016/S2352-3018(17)30066-8

2. Li Z, Purcell DW, Sansom SL, Hayes D, Hall HI. Vital signs: HIV transmission along the continuum of care - United States, 2016. MMWR Morb Mortal Wkly Rep. 2019;68(11):267-272. doi:10.15585/mmwr.mm6811e1

3. HIV Care Continuum. What does the HIV care continuum show? 2020. Available from: https://www.hiv.gov/federal-response/policies-issues /hiv-aids-care-continuum. Accessed May 13, 2020.

4. Ryan White HIV/AIDS Program. Annual Client-Level Data Report. 2018.

5. Kangethe A, Polson M, Lord TC, Evangelatos T, Oglesby A. Real-world health plan data analysis: key trends in medication adherence and overall costs in patients with HIV. J Manag Care Spec Pharm. 2019;25 (1):88-93.

6. Forstein M, Cournos F, Douaihy A, Goodkin K, Wainberg ML, Wapenyi KH. Guideline Watch: Practice Guidelines for the Treatment of Patients with HIV/AIDS. Arlington, VA: American Psychiatric Association; 2006.

7. Paterson DL, Swindells S, Mohr J, et al. Adherence to protease inhibitor therapy and outcomes in patients with HIV infection. Ann Intern Med. 2000;133(1):21-30. doi:10.7326/0003-4819-1331-200007040-00004

8. Wutoh AK, Elekwachi O, Clarke-Tasker V, Daftary M, Powell NJ, Campusano G. Assessment and predictors of antiretroviral adherence in older HIV-infected patients. J Acquir Immune Defic Syndr. 2003;33(Suppl 2):S106-114. doi:10.1097/00126334-20030601200007

9. Davies G, Koenig LJ, Stratford D, et al. Overview and implementation of an intervention to prevent adherence failure among HIV-infected adults initiating antiretroviral therapy: lessons learned from Project HEART. AIDS Care. 2006;18(8):895-903. doi:10.1080/ 09540120500329556

10. Pharmacy Quality Alliance. PQA measure overview; 2019. Available from: https://www.pqaalliance.org/assets/Measures/2019_PQA Measure_Overview.pdf. Accessed April 29, 2020. 
11. Harrigan PR, Hogg RS, Dong WW, et al. Predictors of HIV drug-resistance mutations in a large antiretroviral-naive cohort initiating triple antiretroviral therapy. J Infect Dis. 2005;191(3):339-347. doi:10.1086/427192

12. Meresse M, March L, Kouanfack C, et al. Patterns of adherence to antiretroviral therapy and HIV drug resistance over time in the Stratall ANRS 12110/ESTHER trial in Cameroon. HIV Med. 2014;15(8):478-487. doi:10.1111/hiv.12140

13. US Department of Health and Human Services. Guidelines for the use of antiretroviral agents in adults and adolescents with HIV. Available from: https://aidsinfo.nih.gov/guidelines/html/1/adult-and-adolescentarv/37/whats-new-in-the-guidelines. Accessed January 24, 2020.

14. US Food and Drug Administration. Prescribing information SYMTUZA $^{\circledR} ; 2019$. Available from: https://www.accessdata.fda. gov/drugsatfda_docs/label/2019/210455s004lbl.pdf.

Accessed November 22, 2019.

15. Beer L, Skarbinski J. Adherence to antiretroviral therapy among HIV-infected adults in the United States. AIDS Educ Prev. 2014;26 (6):521-537. doi:10.1521/aeap.2014.26.6.521

16. Kim J, Lee E, Park BJ, Bang JH, Lee JY. Adherence to antiretroviral therapy and factors affecting low medication adherence among incident HIV-infected individuals during 2009-2016: a nationwide study. Sci Rep. 2018;8(1):3133. doi:10.1038/s41598-018-21081-x

17. Simoni JM, Huh D, Wilson IB, et al. Racial/Ethnic disparities in ART adherence in the United States: findings from the MACH14 study. J Acquir Immune Defic Syndr. 2012;60(5):466-472. doi:10.1097/ QAI.0b013e31825db0bd

18. Gross IM, Hosek S, Richards MH, Fernandez MI. Predictors and profiles of antiretroviral therapy adherence among african american adolescents and young adult males living with HIV. AIDS Patient Care STDS. 2016;30(7):324-338. doi:10.1089/apc.2015.0351

19. Altice F, Evuarherhe O, Shina S, Carter G, Beaubrun AC. Adherence to HIV treatment regimens: systematic literature review and meta-analysis. Patient Prefer Adherence. 2019;13:475-490. doi:10.2147/PPA.S192735

20. Chakraborty A, Qato DM, Awadalla SS, Hershow RC, Dworkin MS. Antiretroviral therapy adherence among treatment-naive HIV-infected patients. AIDS. 2020;34(1):127-137. doi:10.1097/ QAD.0000000000002384

21. Chen Y, Chen K, Kalichman SC. Barriers to HIV medication adherence as a function of regimen simplification. Ann Behav Med. 2017;51(1):67-78. doi:10.1007/s12160-016-9827-3

22. Clay PG, Yuet WC, Moecklinghoff $\mathrm{CH}$, et al. A meta-analysis comparing 48-week treatment outcomes of single and multi-tablet antiretroviral regimens for the treatment of people living with HIV. AIDS Res Ther. 2018;15(1):17. doi:10.1186/s12981-018-0204-0

23. Hines DM, Ding Y, Wade RL, Beaubrun A, Cohen JP. Treatment adherence and persistence among HIV-1 patients newly starting treatment. Patient Prefer Adherence. 2019;13:1927-1939. doi:10.2147/PPA. S207908

24. Mohd Salleh NA, Richardson L, Kerr T, et al. A longitudinal analysis of daily pill burden and likelihood of optimal adherence to antiretroviral therapy among people living with HIV who use drugs. J Addict Med. 2018;12(4):308-314. doi:10.1097/ADM.0000000000000403

25. Raffi F, Yazdanpanah Y, Fagnani F, Laurendeau C, Lafuma A, Gourmelen J. Persistence and adherence to single-tablet regimens in HIV treatment: a cohort study from the French National Healthcare Insurance Database. J Antimicrob Chemother. 2015;70(7):2121-2128.

26. Sutton SS, Hardin JW, Bramley TJ, D'Souza AO, Bennett CL. Single- versus multiple-tablet HIV regimens: adherence and hospitalization risks. Am J Manag Care. 2016;22(4):242-248.

27. Yager J, Faragon J, McGuey L, et al. Relationship between single tablet antiretroviral regimen and adherence to antiretroviral and nonantiretroviral medications among veterans' affairs patients with human immunodeficiency virus. AIDS Patient Care STDS. 2017;31 (9):370-376. doi:10.1089/apc.2017.0081
28. Cotte L, Ferry T, Pugliese P, et al. Effectiveness and tolerance of single tablet versus once daily multiple tablet regimens as first-line antiretroviral therapy - Results from a large french multicenter cohort study. PLoS One. 2017;12(2):e0170661. doi:10.1371/journal. pone.0170661

29. Lewis JM, Smith C, Torkington A, et al. Real-world persistence with antiretroviral therapy for HIV in the United Kingdom: a multicentre retrospective cohort study. J Infect. 2017;74(4):401-407. doi:10.1016/j.jinf.2017.01.012

30. Cohen J, Beaubrun A, Bashyal R, Huang A, Li J, Baser O. Realworld adherence and persistence for newly-prescribed HIV treatment: single versus multiple tablet regimen comparison among US medicaid beneficiaries. AIDS Res Ther. 2020;17(1):12. doi:10.1186/ s12981-020-00268-1

31. US Department of Health and Human Services. Human research protections: regulations and policy. Available from: www.hhs.gov/ ohrp/humansubjects/guidance/45cfr46.html\#46.101. Accessed July $15,2020$.

32. American Pharmacists Association. Measuring adherence. Available from: https://www.pharmacist.com/measuring-adherence. Accessed January 24, 2020.

33. Quan H, Li B, Couris CM, et al. Updating and validating the Charlson comorbidity index and score for risk adjustment in hospital discharge abstracts using data from 6 countries. Am J Epidemiol. 2011;173(6):676-682. doi:10.1093/aje/kwq433

34. Hardy H, Luo D, Israel D, Simonson RB, Dunn K. Treatment adherence and virologic response over 48 weeks among patients rapidly initiating darunavir/cobicistat/emtricitabine/tenofovir alafenamide (D/C/F/TAF) in the DIAMOND study [Poster 606]. Presented at: 14th International Conference on HIV Treatment and Prevention Adherence17-19 June 2019; Miami, FL.

35. Sax PE, Meyers JL, Mugavero M, Davis KL, Ross JS. Adherence to antiretroviral treatment and correlation with risk of hospitalization among commercially insured HIV patients in the United States. PLoS One. 2012;7(2):e31591. doi:10.1371/journal.pone.0031591

36. Bourgi K, Rebeiro PF, Turner M, et al. Greater weight gain in treatment naive persons starting dolutegravir-based antiretroviral therapy. Clin Infect Dis. 2019.

37. Karam M, Laurence B, Ricky H, et al. Changes in BMI associated with antiretroviral regimens in treatment-experienced, virologically suppressed individuals living with HIV. Presented at: IDWeek2019; Washington, DC.

38. Kerchberger AM, Sheth AN, Angert CD, et al. Weight gain associated with integrase stand transfer inhibitor use in women. Clin Infect Dis. 2019.

39. Norwood J, Turner M, Bofill C, et al. Brief report: weight gain in persons with HIV switched from efavirenz-based to integrase strand transfer inhibitor-based regimens. J Acquir Immune Defic Syndr. 2017;76(5):527-531. doi:10.1097/QAI.0000000000001525

40. Plankey M, Bacchetti P, Jin C, et al. Self-perception of body fat changes and HAART adherence in the women's interagency HIV study. AIDS Behav. 2009;13(1):53-59. doi:10.1007/s10461-0089444-7

41. Kumar S, Samaras K. The impact of weight gain during HIV treatment on risk of pre-diabetes, diabetes mellitus, cardiovascular disease, and mortality. Front Endocrinol (Lausanne). 2018;9:705. doi: 10.3389/fendo.2018.00705

42. Cantudo-Cuenca MR, Jimenez-Galan R, Almeida-Gonzalez CV, Morillo-Verdugo R. Concurrent use of comedications reduces adherence to antiretroviral therapy among HIV-infected patients. J Manag Care Spec Pharm. 2014;20(8):844-850.

43. Centers for Disease Control and Prevention. HIV surveillance report, 2018 (Preliminary); vol. 30; 2019. Available from: http://www.cdc.gov/ hiv/library/reports/hiv-surveillance.html. Accessed April 29, 2020. 


\section{Publish your work in this journal}

Patient Preference and Adherence is an international, peer-reviewed, open access journal that focusing on the growing importance of patient preference and adherence throughout the therapeutic continuum. Patient satisfaction, acceptability, quality of life, compliance, persistence and their role in developing new therapeutic modalities and compounds to optimize clinical outcomes for existing disease states are major areas of interest for the journal. This journal has been accepted for indexing on PubMed Central. The manuscript management system is completely online and includes a very quick and fair peer-review system, which is all easy to use. Visit http:// www.dovepress.com/testimonials.php to read real quotes from published authors. 